\title{
ROSENLICHT FIELDS
}

\author{
JOHN SHACKELL
}

\begin{abstract}
Let $\phi$ satisfy an algebraic differential equation over $\mathbf{R}$. We show that if $\phi$ also belongs to a Hardy field, it possesses an asymptotic form which must be one of a restricted number of types. The types depend only on the order of the differential equation. For a particular equation the types are still more restricted. In some cases one can conclude that no solution of the given equation lies in a Hardy field, and in others that a particular asymptotic form is the only possibility for such solutions. This therefore gives a new method for obtaining asymptotic solutions of nonlinear differential equations. The techniques used are in part derived from the work of Rosenlicht in Hardy fields.
\end{abstract}

\section{INTRODUCTION}

Let $\mathcal{X}$ be the ring of germs of $\mathscr{C}^{\infty}$ real-valued functions defined on deleted neighbourhoods of infinity in $\mathbf{R}$. A subfield of $\mathcal{X}$ which is closed under differentiation is called a Hardy field (see [3, 7, 1]). If $f$ is a nonzero element of a Hardy field, the requirement that $f$ possess an inverse forces $f$ to be either ultimately positive or ultimately negative. If $g$ is another element we can define $f>g$ to mean that $f-g$ is ultimately positive. Thus a Hardy field possesses a natural ordering which reflects the asymptotic behaviour of the elements.

Hardy showed [5] that one obtains a subfield of $\mathcal{X}$ by closing $\mathbf{R}(x)$ under the operations $f \rightarrow \exp (f)$ and $f \rightarrow \log |f|$ (as well as field operations). This field, which we will denote by $\mathscr{H}$, is clearly closed under differentiation and is thus a Hardy field. Of course it is this example which gave the name to the class.

In [5], Hardy also discussed the representation of asymptotic growth, using iterated exponentials and logarithms, initiated by du Bois-Reymond [4]. Define $e_{0}$ and $l_{0}$ to both be the identity function, $x$, and for $r \geq 1$, let

$$
e_{r}=\exp \left(e_{r-1}\right), \quad l_{r}=\log \left(l_{r-1}\right) .
$$

Here, and throughout the paper, we will use the letter $\varepsilon$ to stand for any element of $\mathcal{X}$ which tends to zero (as $x \rightarrow \infty$ ); so $\varepsilon$ will not necessarily be the same function at each occurrence. The expressions

$$
e_{1}\left(l_{1}^{2}(5+\varepsilon)\right), \quad e_{2}\left(l_{2}(2+\varepsilon)\right), \quad e_{3}\left(l_{3}(1+\varepsilon)\right),
$$

Received by the editors October 16, 1990.

1991 Mathematics Subject Classification. Primary 34E05, 26A12, 68Q40, 12H05, $12 \mathrm{~J} 15$.

Key words and phrases. Hardy fields, asymptotic expansions, orders of growth. 
for example, may each be used to represent the growth (as $x \rightarrow \infty$ ) of elements of $\mathscr{H}$. We note, as Hardy did implicitly, that the expressions in (1) could each describe the growth of the same element of $\mathscr{H}$ but with decreasing precision.

As a still cruder measure of growth, one could ask whether, if $f$ belongs to a Hardy field and tends to infinity, there must exist an $n$ such that

$$
l_{n}<f(x)<e_{n}
$$

for sufficiently large $x$. This is false for arbitrary elements of $\mathcal{X}$ by the theorem of du Bois-Reymond, but holds for elements of $\mathscr{H}$ (see $[4,5])$. In fact, much more is true in $\mathscr{H}$. In [10] an algorithm is given for expressing elements of $\mathscr{H}$ which tend to infinity in the form

$$
e_{s}\left(l_{0}^{p_{0}} \cdots l_{k}^{p_{k}}(c+\varepsilon)\right),
$$

where $p_{0}, \ldots, p_{k}$ and $c$ are constants. Analagous expressions for other elements of $\mathscr{H}$ can similarly be obtained and the $\varepsilon$ can be recursively estimated in like fashion.

In a general Hardy field, (2) may fail for every $n$, as shown in [2]. But for an important subclass, namely the Hardy fields of finite rank [9], one can do much more. Let $\mathscr{F}$ be such a field and let $\phi$ be an element of $\mathscr{F}$ which tends to infinity. Rosenlicht proved in [6] that under these circumstances there exist nonnegative integers $n$ and $m$ such that

$$
\phi=e_{n}\left(l_{m}(1+\varepsilon)\right) \text {. }
$$

As we shall see in the next section, it is an easy consequence of Rosenlicht's work that a similar estimate exists for the $\varepsilon$.

If $\phi$ belongs to a Hardy field and satisfies an algebraic differential equation of order $k$ over $\mathbf{R}$, then $\mathbf{R}\left(\phi, \phi^{\prime}, \ldots, \phi^{(k)}\right)$ is a Hardy field of rank not exceeding $k$ (see [9, Proposition 5]). Hence $\phi$ can be expressed in the form given by (4). From a computational point of view one would like to be able to determine, firstly, whether a given differential equation has some, or all, of its solutions lying in a Hardy field. Secondly, if a solution, $\phi$, does lie in a Hardy field we would like an algorithm to compute its asymptotic form. The form given in (4) will be rather coarse for the purpose (though not without interest) unless we can compute an asymptotic form for the $\varepsilon$.

In $\S 2$ we collect together a number of definitions and results from the papers [6]-[9] of Rosenlicht. These provide the foundation for what follows. In $\S 3$ we take the first step towards strengthening (4). Theorem 5 is the main result here. The heart of the paper lies in $\S 4$. The major results are Theorem 6 and Theorem 7. The techniques used in $\S \S 3$ and 4 are to a certain extent parallel to (and based on) those used by Rosenlicht. However our viewpoint throughout is more computational. In $\S 5$ we show how the estimates of the previous section may be applied to establish possible asymptotic forms for solutions of a given algebraic differential equation or, in some cases, to prove that no nonconstant solution of the equation lies in a Hardy field.

The author would like to thank Max Rosenlicht for providing the original inspiration for this work through his papers [6]-[9], and also for simplifying the proof of Theorem 5 . 


\section{SOME RESULTS OF ROSENLICHT}

In this section we collect together some basic definitions and theorems from [6]-[9]. These will provide a technical foundation for the later sections and also help to motivate them.

Let $\mathscr{F}$ be a Hardy field. We shall generally use $\mathscr{F}^{*}$ to denote nonzero elements of $\mathscr{F}$. If $a$ and $b$ belong to $\mathscr{F}^{*}$, we write $a \asymp b$ if $a(x) / b(x)$ tends to a nonzero, finite limit. Then $\asymp$ is easily seen to be an equivalence relation on $\mathscr{F}^{*}$. We denote the equivalence class of $a$ by $\nu(a)$ and the set of all equivalence classes by $\Upsilon$. The set $\Upsilon$ is an abelian group under the multiplicative operation inherited from $\mathscr{F}^{*}$. Next we write $\nu(a)>\nu(b)$ if $a(x) / b(x) \rightarrow 0$ as $x \rightarrow \infty$. This defines a total ordering on $\Upsilon$ making it an ordered group. We use the notation $a \sim b$ to mean that $a(x) / b(x) \rightarrow 1$. The following is from [7].

Theorem 1 (Rosenlicht). Let $\mathscr{F}$ be a Hardy field. Then there exists a map $\nu$ from $\mathscr{F}^{*}$ onto an ordered abelian group, $\Upsilon$, such that

(1) if $a, b \in \mathscr{F}^{*}$, then $\nu(a b)=\nu(a)+\nu(b)$;

(2) if $a \in \mathscr{F}^{*}$, then $\nu(a) \geq 0$ if and only if $a(x)$ has a finite limit as $x \rightarrow \infty$;

(3) if $a, b \in \mathscr{F}^{*}$, and $a+b \in \mathscr{F}^{*}$, then $\nu(a+b) \geq \min \{\nu(a), \nu(b)\}$ with equality if $\nu(a) \neq \nu(b)$;

(4) if $a, b \in \mathscr{F}^{*}$, and $\nu(a), \nu(b) \neq 0$, then $\nu(a) \geq \nu(b)$ if and only if $\nu\left(a^{\prime}\right) \geq \nu\left(b^{\prime}\right)$

(5) if $a, b \in \mathscr{F}^{*}$ and $\nu(a)>\nu(b) \neq 0$, then $\nu\left(a^{\prime}\right)>\nu\left(b^{\prime}\right)$;

(6) if $a, b \in \mathscr{F}^{*}, a \sim b$, and $\nu(a)(=\nu(b)) \neq 0$, then $a^{\prime} \sim b^{\prime}$.

Let $f$ and $g$ be two infinitely increasing elements of a Hardy field $\mathscr{F}$ (i.e., $f$ and $g$ tend to infinity). We say that $f$ and $g$ are comparable if there exist positive integers $n, m$ such that $f<g^{n}$ and $g<f^{m}$. The notion may be extended to elements tending to zero and to minus infinity by specifying that $f$ and $f^{-1}$ be comparable, and likewise $f$ and $-f$, for all infinitely-increasing $f$ in $\mathscr{F}$. We also specify that any two elements which tend to a nonzero finite limit be comparable. (This is a trivial, but notationally convenient extension of Rosenlicht's definition.) Comparability is then an equivalence relation on $\mathscr{F}^{*}$ and the equivalence classes will be called comparability classes. We write $\gamma(f)$ for the comparability class of $f$.

If $f$ and $g$ are infinitely-increasing elements of $\mathscr{F}$, we write $\gamma(f)>\gamma(g)$ if $f>g^{n}$ for all $n \in \mathbf{N}$; it is easy to see that this only depends on the comparability classes. We also specify that $\nu^{-1}(0)$ be the smallest comparability class. Thus $\gamma$ is defined on the whole of $\mathscr{F}^{*}$ and the comparability classes are totally ordered. The number of different comparability classes of $\mathscr{F}^{*}$ minus one (or equivalently the number of comparability classes of $\mathscr{F} \backslash \nu^{-1}(0)$ ) is called the rank of $\mathscr{F}$. The following is taken from [9].

Proposition 1 (Rosenlicht). Let $\mathscr{F} \subset \mathscr{G}$ be Hardy fields and let $r$ be the transcendence degree of $\mathscr{G}$ over $\mathscr{F}$. Then rank $\mathscr{G} \leq$ rank $\mathscr{F}+r$. In particular, if $\phi$ belongs to a Hardy field and satisfies an algebraic differential equation of order $k$ over $\mathscr{F}$, then the rank of $\mathscr{F}\left(\phi, \phi^{\prime}, \ldots, \phi^{(k)}\right)$ is at most $k+$ rank $\mathscr{F}$.

The next result is concerned with the adjoining elements to a Hardy field. It comes from [7]. 
Theorem 2 (Rosenlicht). Let $\mathscr{F}$ be a Hardy field and let $f(Y), g(Y) \in \mathscr{F}[Y]$, where $Y$ is an indeterminate. Let $y \in \mathcal{X}$ and suppose $y(x)$ satisfies $y^{\prime}(x)=$ $f(y(x)) / g(y(x))$ on a positive half line on which $g(y(x))$ is nonzero. Then $\mathscr{F}(y)$ is a Hardy field.

It is worth remarking that the rank of $\mathscr{F}(y)$ does not exceed the rank of $\mathscr{F}$ by more than one. This is a direct consequence of Proposition 1 .

It follows from Theorem 2 that if $\phi$ belongs to a Hardy field $\mathscr{F}$ then $\mathscr{F}$ may be enlarged (if necessary) to a Hardy field containing $\log |\phi|$ and $\exp (\phi)$. Moreover, since $1 \in \mathscr{F}$, we may enlarge $\mathscr{F}$ to contain $x, \log x$, $\exp (x)$, etc. So if $\phi$ belongs to a Hardy field, comparisons like $e_{r}(\phi)<l_{n}$ make sense. In fact we shall frequently write such inequalities without making specific reference to the Hardy field which contains the exponentials and logarithms.

The following result is also essentially due to Rosenlicht.

Theorem 3 (Rosenlicht). Let $\mathscr{F}$ be a Hardy field of finite rank $r$ and let $\phi$ be an infinitely-increasing element of $\mathscr{F}$. Then there is an integer $s$ with $|s| \leq r$ such that for all integers $n>r$

$$
\phi=e_{n}\left(l_{n-s}(1+\varepsilon)\right) .
$$

Moreover, $\varepsilon$ belongs to a Hardy field of rank no greater than $r+2 n-s+1$ and so a similar expression exists for $\varepsilon$.

Proof of Theorem 3. That $\phi$ can be expressed in the form (5) is just Theorem 1 of [6]. Then $\varepsilon=l_{n}(\phi) / l_{n-s}-1$ and so $\varepsilon$ belongs to a Hardy field by Theorem 2. The statement about the rank of this Hardy field then follows from Proposition 5 of [9] as indicated above. This completes the proof.

Theorem 3 is interesting but in some ways is less than one would desire. The estimate on the right of (5) is rather crude, corresponding only to the coarsest of the forms in (1). Moreover, the greater the rank, the worse the estimate. So the estimate for $\varepsilon$ may be significantly less accurate even than that for $\phi$. If we knew that $l_{n}(\phi)$ belonged to $\mathscr{F}$, matters would be much improved. Proposition 6 of [9] gives some hope for this because it asserts that if $f \in \mathscr{F}$ and $f \rightarrow \infty$, then $\mathscr{F}$ contains an element asymptotic to $\log f$, provided that $\mathscr{F}$ contains elements of comparability class smaller than that of $f$. But how do we know that $\mathscr{F}$ does contain such elements? One of the main results of the present paper is concerned with giving a much sharper version of (5) with generally a corresponding improvement in the estimate for the $\varepsilon$.

Next let $\mathbf{Q}$ denote the field of rational numbers and let $\mathscr{F} \subset \mathscr{G}$ be two Hardy fields. There is a natural, order-preserving embedding of ordered abelian groups $\nu\left(\mathscr{F}^{*}\right) \hookrightarrow \nu\left(\mathscr{G}^{*}\right)$. If $\mathscr{G}$ is taken to include all $n$th roots of positive elements of $\mathscr{F}$, then each element of $\nu\left(\mathscr{F}^{*}\right)$ is divisible in $\nu\left(\mathscr{G}^{*}\right)$ by each $n \neq 0$, and so $\nu\left(\mathscr{F}^{*}\right)$ is a subgroup of an ordered vector space over $\mathbf{Q}$. The dimension of the Q-vector space generated by $\nu\left(\mathscr{F}^{*}\right)$ is called the rational rank of $\mathscr{F}$ [8].

The rational rank of $\mathscr{F}$ is at least the rank of $\mathscr{F}$ and is no more than the transcendence degree of $\mathscr{F}$ over its field of constants. Moreover, if $\phi$ satisfies an algebraic differential equation of order $k$ over $\mathscr{F}$ then the rational rank of $\mathscr{F}\left(\phi, \phi^{\prime}, \ldots, \phi^{(n)}\right)$ is not greater than $k$ plus the rational rank of $\mathscr{F}$. These results and also the following are from [8]. 
Theorem 4 (Rosenlicht). Let $\mathscr{F}$ be a Hardy field which contains $\boldsymbol{R}$. There is a smallest Hardy field $\mathscr{G} \supset \mathscr{F}$ such that $|t|^{c} \in \mathscr{G}$ whenever $t \in \mathscr{G}^{*}$ and $c \in \mathbf{R}$. Moreover, rank $\mathscr{F} \leq$ rank $\mathscr{G} \leq$ rational rank $\mathscr{F}$.

Since we shall often need to take real powers of elements, Hardy fields of finite rank which contain $\mathbf{R}$ and have the property that $|t|^{c}$ is an element whenever $t$ is a nonzero element and $c \in \mathbf{R}$, will be of particular importance to us. It seems appropriate to call such objects Rosenlicht fields. It is clear from the above that if $\phi$ belongs to a Hardy field and satisfies an algebraic differential equation over a Rosenlicht field then $\phi$ belongs to a Rosenlicht field.

\section{ASYMPTOTIC GROWTH}

Our principal aim in this section is to establish a stronger version of Theorem 3. We begin with two techrical lemmas.

Lemma 1. Let $\mathscr{F}$ be a Hardy field and let $a$ and $b$ belong to $\mathscr{F}^{*}$ with $\nu(a)$ and $\nu(b)$ both nonzero. Then

(i) $\nu\left(a^{\prime} / a\right)=\nu\left(b^{\prime} / b\right)$ if and only if $\gamma(a)=\gamma(b)$,

(ii) $\nu\left(a^{\prime} / a\right)>\nu\left(b^{\prime} / b\right)$ if and only if $\gamma(a)<\gamma(b)$.

Proof of Lemma 1. That $\nu\left(a^{\prime} / a\right)=\nu\left(b^{\prime} / b\right)$ implies $\gamma(a)=\gamma(b)$ is just Proposition 4 of [9]. Suppose then that $\gamma(a)=\gamma(b)$. We may take $a$ and $b$ to be infinitely-increasing and then there is an $n \in \mathbf{N}$ such that $a<b^{n}$. Hence $\log a<n \log b$ and so $\nu(\log a) \geq \nu(\log b)$. It then follows from Theorem 1(4) that $\nu\left(a^{\prime} / a\right) \geq \nu\left(b^{\prime} / b\right)$. Since $a$ and $b$ are interchangeable in the above argument, $\nu\left(a^{\prime} / a\right)=\nu\left(b^{\prime} / b\right)$.

Now suppose that $\gamma(a)<\gamma(b)$. We can deduce, as above, that $\nu\left(a^{\prime} / a\right) \geq$ $\nu\left(b^{\prime} / b\right)$. But $\nu\left(a^{\prime} / a\right)$ and $\nu\left(b^{\prime} / b\right)$ cannot be equal, since then $\gamma(a)=\gamma(b)$. So $\nu\left(a^{\prime} / a\right)>\nu\left(b^{\prime} / b\right)$. Conversely if $\nu\left(a^{\prime} / a\right)>\nu\left(b^{\prime} / b\right)$, then $\gamma(a)=\gamma(b)$ and $\gamma(a)>\gamma(b)$ give contradictions by (i) and the part of (ii) already established, respectively. Hence $\gamma(a)<\gamma(b)$ and this completes the proof.

Our next result is in effect a strengthening of Theorem 3. At first sight, the gain is small, but it is important for what follows.

Theorem 5. Let $\phi$ be an infinitely-increasing element of a Rosenlicht field $\mathscr{F}$. Then there exist nonnegative integers $s$ and $m$, a positive real number $d$, and an element $\phi_{1}$ of $\mathcal{X}$, such that

(i) $\phi=e_{s}\left(l_{m}^{d} \phi_{1}\right)$,

(ii) $\gamma\left(\phi_{1}\right)<\gamma\left(l_{m}\right)$,

(iii) if $s>0, m>0$, and $d=1$, then $\nu\left(\phi_{1}\right) \neq 0$,

(iv) $\phi_{1}$ is an element of the Rosenlicht field generated by $x, l_{1}, l_{2}, \ldots, l_{m}$, $l_{1}(\phi), \ldots, l_{s}(\phi)$, and $\mathscr{F}$.

Proof of Theorem 5. The following proof is due to Rosenlicht. It replaces a longer and clumsier proof by the author.

It follows from Theorem 3 that there exists an element $\phi_{1}$ in some Hardy field such that $\phi=e_{s}\left(l_{m}^{d} \phi_{1}\right)$ with $m, s \in \mathbf{N}, d \in \mathbf{R}^{+}$, and $\gamma\left(\phi_{1}\right)<\gamma\left(l_{m}\right)$. Take such an expression with $s$ minimal. If $\nu\left(\phi_{1}\right)=0$, then $\phi_{1} \sim c$ for some 
constant $c$. If in addition $s>0, m>0$, and $d=1$, then

$$
\phi=e_{s-1}\left(\left(e^{l_{m}}\right)^{\phi_{1}}\right)=e_{s-1}\left(l_{m-1}^{c} l_{m-1}^{\phi_{1}-c}\right)
$$

and $\gamma\left(l_{m-1}^{\phi_{1}-c}\right)<\gamma\left(l_{m-1}\right)$. But this contradicts the minimality of $s$. We have therefore established (i), (ii), and (iii). Since (iv) is an easy consequence of (i), this suffices to prove Theorem 5 .

\section{RANK BOUNDS AND NESTED FORMS}

In Theorem 5 we have not given any bounds for $m$ and $s$ in terms of the rank of $\mathscr{F}$. Of course the bounds of Theorem 3 also apply here. However we shall establish a much better bound in this section. Secondly we need more information about $\phi_{1}$. Ideally we would like $\phi_{1}$ to belong to $\mathscr{F}$. What we can show, and for our present purpose it is nearly as good, is that $\mathscr{F}$ contains an element asymptotic to $\phi_{1}$.

It will be important to be able to compare the growths of iterated exponentials and this is the purpose of the next lemma.

Lemma 2. Let $f$ and $g$ be infinitely-increasing elements of a Hardy field and suppose, without loss of generality, that $f>g$. Suppose also that $n \geq 1$ and write $h_{n}=e_{n}(f)-e_{n}(g)$ and $H_{n}=(f-g) e_{1}(g) e_{2}(g) \cdots e_{n}(g)$. Then

(i) $h_{n} \rightarrow \infty$ if and only if $H_{n} \rightarrow \infty$,

(ii) $h_{n} \rightarrow 0$ if and only if $H_{n} \rightarrow 0$ and in this case $h_{n} \sim H_{n}$,

(iii) $h_{n}$ tends to a positive finite limit if and only if $H_{n}$ does so, and here also $h_{n} \sim H_{n}$.

Proof of Lemma 2. The proof is by induction. We have

$$
h_{1}=e_{1}(f)-e_{1}(g)=\left(e_{1}(f-g)-1\right) e_{1}(g) .
$$

If $f-g \rightarrow 0$ then $e_{1}(f-g)-1 \sim f-g$, and so $h_{1} \sim H_{1}$. On the other hand, if $f-g$ does not tend to zero, $H_{1}$ must tend to infinity and, since $e_{1}(f-g)-1>(f-g)$, so must $h_{1}$. Thus the result holds when $n=1$.

Suppose then that the result holds for a particular value, $n$. Then

$$
\begin{aligned}
h_{n+1} & =e_{n+1}(f)-e_{n+1}(g)=\left\{e_{1}\left(e_{n}(f)-e_{n}(g)\right)-1\right\} e_{n+1}(g) \\
& =\left\{e_{1}\left(h_{n}\right)-1\right\} e_{n+1}(g) .
\end{aligned}
$$

As before, $e_{1}\left(h_{n}\right)-1>h_{n}$ and if $h_{n} \rightarrow 0$, then $e_{1}\left(h_{n}\right)-1 \sim h_{n} \sim H_{n}$, by the induction hypothesis. So, if $h_{n} \rightarrow 0$, then $h_{n+1} \sim H_{n} e_{n+1}(g)=H_{n+1}$. If $h_{n} \nrightarrow 0$, then $H_{n} \nrightarrow 0$ by the induction hypothesis and then both $h_{n+1}$ and $H_{n+1}$ tend to infinity. This completes the proof of Lemma 2.

Our next lemma is concerned with the order relation between the comparability classes of two elements each of the form $e_{s}\left(l_{m}^{d} \phi_{1}\right)$.

Lemma 3. Let $\phi_{1}$ and $\phi_{2}$ belong to a Hardy field, let $m . n, s$, and t be nonnegative integers, and let $c$ and $d$ be positive real numbers. Suppose that $\gamma\left(\phi_{1}\right)<\gamma\left(l_{m}\right)$ and $\gamma\left(\phi_{2}\right)<\gamma\left(l_{n}\right)$. Suppose also that if $c=1$ then $\nu\left(\phi_{2}\right) \neq 0$ and if $d=1$ then $\nu\left(\phi_{1}\right) \neq 0$. Then

(I) $\gamma\left(e_{s}\left(l_{m}^{d} \phi_{1}\right)\right)=\gamma\left(e_{t}\left(l_{n}^{c} \phi_{2}\right)\right)$ if and only if either

(i) $s=t=0$ and $m=n$, or 
(ii) $s=t=1, m=n, c=d$, and $\nu\left(\phi_{1}\right)=\nu\left(\phi_{2}\right)$, or

(iii) $s=t \geq 2, m=n, c=d$, and

$$
H=\left(\phi_{1}-\phi_{2}\right) l_{n}^{c} e_{1}\left(l_{n}^{c} \phi_{2}\right) e_{2}\left(l_{n}^{c} \phi_{2}\right) \ldots e_{s-2}\left(l_{n}^{c} \phi_{2}\right)
$$

has a finite limit.

(II) $\gamma\left(e_{s}\left(l_{m}^{d} \phi_{1}\right)\right)>\gamma\left(e_{t}\left(l_{n}^{c} \phi_{2}\right)\right)$ if and only if either

(i) $s-m>t-n$, or

(ii) $s-m=t-n, s>t$, and either $d>1$, or $\phi_{1} \rightarrow \infty$ and $d=1$ or

(iii) $s-m=t-n, s<t$, and either $c<1$ or $\phi_{2} \rightarrow 0$ and $c=1$, or

(iv) $s=t \geq 1, m=n$, and either $d>c$ or $\phi_{1} / \phi_{2} \rightarrow \infty$ and $d=c$, or

(v) $s=t \geq 2, m=n, d=c$, and $H \rightarrow \infty$, where $H$ is as given in (I)(iii).

Proof of Lemma 3. We prove the sufficiency of the conditions first starting wth (II)(i). Suppose $s-m>t-n$ and that $s \geq t$. Then $m<n+s-$ $t$ and so $\gamma\left(l_{m}\right)>\gamma\left(l_{n+s-t}\right)$. Hence $\gamma\left(l_{s}\left(e_{s}\left(l_{m}^{d} \phi_{1}\right)\right)\right)>\gamma\left(l_{s}\left(e_{t}\left(l_{n}^{c} \phi_{2}\right)\right)\right)$ and so $\gamma\left(e_{s}\left(l_{m}^{d} \phi_{1}\right)\right)>\gamma\left(e_{t}\left(l_{n}^{c} \phi_{2}\right)\right)$. If $s<t$, then $m+t-s<n$ and so $\gamma\left(l_{t}\left(e_{s}\left(l_{m}^{d} \phi_{1}\right)\right)\right)>$ $\gamma\left(l_{t}\left(e_{t}\left(l_{n}^{c} \phi_{2}\right)\right)\right)$. Hence $\gamma\left(e_{s}\left(l_{m}^{d} \phi_{1}\right)\right)>\gamma\left(e_{t}\left(l_{n}^{c} \phi_{2}\right)\right)$ in this case also. Thus (II)(i) is sufficient.

Now suppose $s-m=t-n$ and $s>t$. Then $\nu\left(l_{s}\left(e_{t}\left(l_{n}^{c} \phi_{2}\right)\right)\right)=\nu\left(l_{n+s-t}\right)=$ $\nu\left(l_{m}\right)$. Therefore

$$
\nu\left(\frac{l_{s}\left(e_{s}\left(l_{m}^{d} \phi_{1}\right)\right)}{l_{s}\left(e_{t}\left(l_{n}^{c} \phi_{2}\right)\right)}\right)=\nu\left(\frac{l_{m}^{d} \phi_{1}}{l_{m}}\right) .
$$

However, $l_{m}^{d-1} \phi_{1}$ tends to infinity if $d>1$ or $d=1$ and $\phi_{1} \rightarrow \infty$. Since $s \geq 1$, this will entail that $\gamma\left(e_{s}\left(l_{m}^{d} \phi_{1}\right)\right)>\gamma\left(e_{t}\left(l_{n}^{c} \phi_{2}\right)\right)$ and so (II)(ii) is sufficient. Furthermore, $l_{m}^{d-1} \phi_{1}$ tends to zero if $d<1$ or $d=1$ and $\phi_{1} \rightarrow 0$ and then $\gamma\left(e_{s}\left(l_{m}^{d} \phi_{1}\right)\right)<\gamma\left(e_{t}\left(l_{n}^{c} \phi_{2}\right)\right)$. On interchanging $e_{s}\left(l_{m}^{d} \phi_{1}\right)$ and $e_{t}\left(l_{n}^{c} \phi_{2}\right)$ we see that (II)(iii) is sufficient also.

Next suppose that $s=t$ and $m=n$. If $s=0$, then $e_{s}\left(l_{m}^{d} \phi_{1}\right)=l_{m}^{d} \phi_{1}$ and $e_{t}\left(l_{n}^{c} \phi_{2}\right)=l_{m}^{c} \phi_{2}$. Hence $(\mathrm{I})(\mathrm{i})$ is sufficient. If $s \geq 1$, then

$$
\frac{l_{s}\left(e_{s}\left(l_{m}^{d} \phi_{1}\right)\right)}{l_{s}\left(e_{t}\left(l_{n}^{c} \phi_{2}\right)\right)}=l_{m}^{d-c} \phi_{1} \phi_{2}^{-1} \text {. }
$$

The right-hand side of (7) tends to infinity if $d>c$ or if $d=c$ and $\phi_{1} / \phi_{2} \rightarrow$ $\infty$. Since $s \geq 1$, this shows that (II)(iv) is sufficient. If $s=1, c=d$, and $\nu\left(\phi_{1}\right)=\nu\left(\phi_{2}\right)$, then the right-hand side of (7) tends to a nonzero, finite limit and hence $\gamma\left(e_{1}\left(l_{m}^{d} \phi_{1}\right)\right)=\gamma\left(e_{1}\left(l_{n}^{c} \phi_{2}\right)\right)$. Thus (I)(ii) is sufficient.

Finally consider the case when $s=t \geq 2, n=m$, and $c=d$. Then

$$
\frac{l_{1}\left(e_{s}\left(l_{m}^{d} \phi_{1}\right)\right)}{l_{1}\left(e_{t}\left(l_{n}^{c} \phi_{2}\right)\right)}=\frac{e_{s-1}\left(l_{n}^{c} \phi_{1}\right)}{e_{s-1}\left(l_{n}^{c} \phi_{2}\right)}=e_{1}\left(e_{s-2}\left(l_{n}^{c} \phi_{1}\right)-e_{s-2}\left(l_{n}^{c} \phi_{2}\right)\right) \text {. }
$$

If $s=2$, the expression on the right of $(8)$ is $e_{1}\left(l_{n}^{c}\left(\phi_{1}-\phi_{2}\right)\right)$ which tends to infinity if $l_{n}^{c}\left(\phi_{1}-\phi_{2}\right) \rightarrow \infty$ and to zero if $l_{n}^{c}\left(\phi_{1}-\phi_{2}\right) \rightarrow-\infty$; otherwise it tends to a nonzero finite limit. It follows that (II)(v) and (I)(iii) are sufficient when $s=2$.

If $s>2$, we apply Lemma 2 to the right-hand side of (8), giving that $l_{1}\left(e_{s}\left(l_{m}^{d} \phi_{1}\right)\right) / l_{1}\left(e_{t}\left(l_{n}^{c} \phi_{2}\right)\right)$ tends to infinity if $H$ does and to a nonzero finite limit 
if $H$ tends to a finite limit. This shows that (II)(v) and (I)(iii) are sufficient. Thus all the conditions are sufficient to give the desired conclusion.

If in (II) we interchange $e_{s}\left(l_{m}^{d} \phi_{1}\right)$ and $e_{t}\left(l_{n}^{c} \phi_{2}\right)$ we obtain an analogous set of conditions, which we will refer to as (III), for $\gamma\left(e_{s}\left(l_{m}^{d} \phi_{1}\right)\right)<\gamma\left(e_{t}\left(l_{n}^{c} \phi_{2}\right)\right)$. Now since the cases $d=1, \nu\left(\phi_{1}\right)=0$ and $c=1, \nu\left(\phi_{2}\right)=0$ are specifically excluded, a routine check shows that (I), (II), and (III) between them exhaust the possibilities. It then follows that (I) and (II) are necessary. For example, if $\gamma\left(e_{s}\left(l_{m}^{d} \phi_{1}\right)\right)=\gamma\left(e_{t}\left(l_{n}^{c} \phi_{2}\right)\right)$ then one of the conditions (I) must hold, for if not then one of (II) or one of (III) would have to hold, giving a contradiction. This completes the proof of Lemma 3.

The following result is proved in a similar fashion, though it is considerably simpler.

Lemma 4. Let $\phi_{1}$ belong to a Hardy field. Let $s, m$, and $j$ be nonnegative integers and let $d$ be a positive real number. Suppose that if $d=1$ then $\nu\left(\phi_{1}\right) \neq 0$. Then $\gamma\left(e_{s}\left(l_{m}^{d} \phi_{1}\right)\right)=\gamma\left(l_{j}\right)$ if and only if $s=0$ and $m=j$, while $\gamma\left(e_{s}\left(l_{m}^{d} \phi_{1}\right)\right)>\gamma\left(l_{j}\right)$ if and only if one of the three following conditions holds:

(i) $m<s+j$;

(ii) $m=s+j, s>0$, and $d>1$;

(iii) $m=s+j, s>0, d=1$, and $\phi_{1} \rightarrow \infty$.

The next result is perhaps the centre of gravity of the paper; it assumes the existence of an element of the type given by Theorem 5 and deduces the existence of various comparability classes.

Theorem 6. Let $\mathscr{F}$ be a Rosenlicht field and let $\phi \in \mathscr{F}$. Suppose that $\phi=$ $e_{s}\left(l_{m}^{d} \phi_{1}\right)$, where $\gamma\left(\phi_{1}\right)<\gamma\left(l_{m}\right), d \in \mathbf{R}^{+}$, and if $s>0, m>0$, and $d=1$, then $\nu\left(\phi_{1}\right) \neq 0$. Then there exists an integer $\sigma \geq 0$ and elements $\Gamma_{0}, \Gamma_{1}, \ldots, \Gamma_{\sigma}$ of $\mathscr{F}$ with the following properties:

(i) $\gamma\left(\Gamma_{i}\right)>\gamma\left(\Gamma_{i+1}\right)$ for $i=0, \ldots, \sigma-1$.

(ii) If $d \neq 1$ or if $s=0$, then $\sigma=s+m+1$; otherwise $\sigma=s+m$.

(iii) Each $\Gamma_{i}$ may be expressed as a rational function of a finite set of real powers of $\phi, \phi^{\prime}, \ldots, \phi^{(i)}$ with real coefficients. Conversely, $\phi^{(i)}$ may be similarly expressed as a rational function of a finite set of real powers of $\Gamma_{0}, \Gamma_{1}, \ldots, \Gamma_{i}$. Both of these rational functions are computable.

(iv) The set $\left\{\gamma\left(\Gamma_{0}\right), \ldots, \gamma\left(\Gamma_{\sigma}\right)\right\}$ contains the following comparability classes: if $s>0$, it contains $\gamma\left(e_{1}\left(l_{m}^{d} \phi_{1}\right)\right), \ldots, \gamma\left(e_{s}\left(l_{m}^{d} \phi_{1}\right)\right)$; if $m>0$, it contains $\gamma\left(l_{0}\right), \ldots, \gamma\left(l_{m-1}\right)$; it contains $\gamma\left(\phi_{1}\right)$, and if $d \neq 1$ or if $s=0$, it also contains $\gamma\left(l_{m}\right)$.

Proof of THEOREM 6. The proof will be divided into a number of cases. There is however some common ground between them. In [9], in particular in Theorem 1 and Proposition 6, a similar technique is used.

For any pair of infinitely-increasing elements $f$ and $g$ of $\mathscr{F}$, we write $f \approx g$ if for every real $\delta>0, g^{1-\delta}<f<g^{1+\delta}$. Clearly $f \approx g$ if and only if $\log f \sim \log g$, and then, provided that $\nu(f) \neq 0$, it follows that $f^{\prime} / f \sim g^{\prime} / g$ by Theorem 1(6). A generic method of producing suitable $\Gamma_{i}$ 's is then as follows: 
Suppose we already have $\Gamma_{j}$ with $\Gamma_{j} \approx e_{t}\left(l_{m}^{d} \phi_{1}\right), t \geq 2$. Then $\log \Gamma_{j} \sim$ $e_{t-1}\left(l_{m}^{d} \phi_{1}\right)$, and so

$$
\begin{aligned}
\frac{\Gamma_{j}^{\prime}}{\Gamma_{j}} & \sim\left(e_{t-1}\left(l_{m}^{d} \phi_{1}\right)\right)^{\prime} \\
& =e_{t-1}\left(l_{m}^{d} \phi_{1}\right) \cdots e_{1}\left(l_{m}^{d} \phi_{1}\right) l_{0}^{-1} l_{1}^{-1} \cdots l_{m-1}^{-1}\left(l_{m}^{d-1} \phi_{1} d+l_{m}^{d} \phi_{1}^{\prime}\right) .
\end{aligned}
$$

Since $\gamma\left(\phi_{1}\right)<\gamma\left(l_{m}\right)$, Lemma 1 and Theorem $1(\mathrm{i})$ imply that $\nu\left(\phi_{1}^{\prime} / \phi_{1}\right)>$ $\nu\left(l_{m}^{-1}\right)$, and so from (9)

$$
\frac{\Gamma_{j}^{\prime}}{\Gamma_{j}} \sim e_{t-1}\left(l_{m}^{d} \phi_{1}\right) \cdots e_{1}\left(l_{m}^{d} \phi_{1}\right) l_{0}^{-1} l_{1}^{-1} \cdots l_{m-1}^{-1} l_{m}^{d-1} \phi_{1} d .
$$

Suppose in addition that we have already defined elements $\eta_{0}, \ldots, \eta_{k}$ of $\mathscr{F}$ with $\eta_{i} \sim l_{i}^{-1}$ for $i=0, \ldots, k$ and that $\gamma\left(\eta_{k}\right)>\gamma\left(e_{t-1}\left(l_{m}^{d} \phi_{1}\right)\right)$. Then we let $\Gamma_{j+1}=\Gamma_{j}^{\prime} \Gamma_{j}^{-1} \eta_{0}^{-1} \cdots \eta_{k}^{-1}$. It follows that either $\Gamma_{j+1} \approx l_{k+1}^{-1}$ if $\gamma\left(l_{k+1}\right)>$ $\gamma\left(e_{t-1}\left(l_{m}^{d} \phi_{1}\right)\right)$ or $\Gamma_{j+1} \approx e_{t-1}\left(l_{m}^{d} \phi_{1}\right)$ if $\gamma\left(l_{k+1}\right)<\gamma\left(e_{t-1}\left(l_{m}^{d} \phi_{1}\right)\right)$. Note that the latter two comparability classes cannot be equal because of Lemma 4 (the case $m=0$ is trivial). If $\Gamma_{j+1} \approx l_{k+1}^{-1}$, then $\Gamma_{j+1}^{\prime} / \Gamma_{j+1} \sim-l_{0}^{-1} l_{1}^{-1} \cdots l_{k+1}^{-1}$ and so we take $\eta_{k+1}=-\Gamma_{j+1}^{\prime} \Gamma_{j+1}^{-1} \eta_{0}^{-1} \cdots \eta_{k}^{-1}$; then $\eta_{k+1} \sim l_{k+1}^{-1}$. We then take $\Gamma_{j+2}=\Gamma_{j+1} \eta_{k+1}^{-1}$ so that either $\Gamma_{j+2} \approx e_{t-1}\left(l_{m}^{d} \phi_{1}\right)$ or $\Gamma_{j+2} \approx l_{k+2}^{-1}$. If, on the other hand, $\Gamma_{j+1} \approx e_{t-1}\left(l_{m}^{d} \phi_{1}\right)$ and $t>2$, we take $\Gamma_{j+2}=-\Gamma_{j+1}^{\prime} \Gamma_{j+1}^{-1} \eta_{0}^{-1} \cdots \eta_{k}^{-1}$ and, since $\gamma\left(l_{k}\right)>\gamma\left(e_{t-1}\left(l_{m}^{d} \phi_{1}\right)\right)$, it follows that $\gamma\left(l_{k+1}\right)>\gamma\left(e_{t-2}\left(l_{m}^{d} \phi_{1}\right)\right)$ and hence that $\Gamma_{j+2} \approx l_{k+1}^{-1}$. Then $\eta_{k+1}$ is defined as before.

In practice we shall generally use a slightly different construction which makes better use of elements already defined. In addition 'end' cases will need special attention. We give details of the cases $s>m>0$ and $m>s>0$ only. Other cases are similar to, or degenerate versions of, one of these. For full details see [11].

We shall write $f^{\Delta}$ for the logarithmic derivative of an element $f$ of $\mathcal{X}$, so that $f^{\Delta}=f^{\prime} / f$.

Case 1: $s>m>0$. By Lemma 4, $\gamma(\phi)=\gamma\left(e_{s}\left(l_{m}^{d} \phi_{1}\right)\right)>\gamma\left(l_{0}\right)$. We therefore take $\Gamma_{0}=\phi$. For $i=0, \ldots, s-m-1$, we take $\Gamma_{i+1}=\Gamma_{i}^{\Delta}$. Suppose $0 \leq i \leq s-m-2$ and $\Gamma_{i} \approx e_{s-i}\left(l_{m}^{d} \phi_{1}\right)$; of course, for $i=0$, we know this. By (10),

$$
\Gamma_{i+1} \sim e_{s-i-1}\left(l_{m}^{d} \phi_{1}\right) \cdots e_{1}\left(l_{m}^{d} \phi_{1}\right) l_{0}^{-1} \cdots l_{m-1}^{-1} l_{m}^{d-1} \phi_{1} d,
$$

and, by Lemma 4, $\gamma\left(e_{s-i-1}\left(l_{m}^{d} \phi_{1}\right)\right)>\gamma\left(l_{0}\right)$, since $s-i-1>m$. Hence $\Gamma_{i+1} \approx$ $e_{s-i-1}\left(l_{m}^{d} \phi_{1}\right)$ and so, by induction, $\Gamma_{i} \approx e_{s-i}\left(l_{m}^{d} \phi_{1}\right)$ for $i=0, \ldots, s-m-1$. From (10) again we have

$$
\Gamma_{s-m} \sim e_{m}\left(l_{m}^{d} \phi_{1}\right) \cdots e_{1}\left(l_{m}^{d} \phi_{1}\right) l_{0}^{-1} \cdots l_{m-1}^{-1} l_{m}^{d-1} \phi_{1} d .
$$

Case 1a: $d>1$ or $d=1$ and $\phi_{1} \rightarrow \infty$. In this case, Lemma 4 implies that $\Gamma_{s-m} \approx e_{m}\left(l_{m}^{d} \phi_{1}\right)$. We take $\Gamma_{s-m+1}=\Gamma_{s-m}^{\Delta}$ so that

$$
\Gamma_{s-m+1} \sim e_{m-1}\left(l_{m}^{d} \phi_{1}\right) \cdots e_{1}\left(l_{m}^{d} \phi_{1}\right) l_{0}^{-1} \cdots l_{m-1}^{-1} l_{m}^{d-1} \phi_{1} d .
$$


From Lemma 4, $\Gamma_{s-m+1} \approx l_{0}^{-1}$. It follows that $\Gamma_{s-m+1}^{\Delta} \sim-l_{0}^{-1}$. Hence we take $\Gamma_{s-m+2}=-\Gamma_{s-m+1} / \Gamma_{s-m+1}^{\Delta}$. Then, from (12),

$$
\Gamma_{s-m+2} \sim e_{m-1}\left(l_{m}^{d} \phi_{1}\right) \cdots e_{1}\left(l_{m}^{d} \phi_{1}\right) l_{\mathrm{i}}^{-1} \cdots l_{m-1}^{-1} l_{m}^{d-1} \phi_{1} d \approx e_{m-1}\left(l_{m}^{d} \phi_{1}\right),
$$

by Lemma 4 .

Suppose $0<i<m-1$ and that $\Gamma_{s-m+2 i-1}$ and $\Gamma_{s-m+2 i}$ have been defined with $\Gamma_{s-m+2 i-1} \approx l_{i-1}^{-1}$ and $\Gamma_{s-m+2 i} \approx e_{m-i}\left(l_{m}^{d} \phi_{1}\right)$. Then

$$
\Gamma_{s-m+2 i-1}^{\Delta} \sim-l_{0}^{-1} l_{1}^{-1} \cdots l_{i-1}^{-1}
$$

and

$$
\Gamma_{s-m+2 i}^{\Delta} \sim e_{m-i-1}\left(l_{m}^{d} \phi_{1}\right) \cdots e_{1}\left(l_{m}^{d} \phi_{1}\right) l_{0}^{-1} \cdots l_{m-1}^{-1} l_{m}^{d-1} \phi_{1} d .
$$

We take

$$
\Gamma_{s-m+2 i+1}=-\Gamma_{s-m+2 i}^{\Delta} / \Gamma_{s-m+2 i-1}^{\Delta} .
$$

It then follows from (13) and (14) that

$$
\Gamma_{s-m+2 i+1} \sim e_{m-i-1}\left(l_{m}^{d} \phi_{1}\right) \cdots e_{1}\left(l_{m}^{d} \phi_{1}\right) l_{i}^{-1} \cdots l_{m-1}^{-1} l_{m}^{d-1} \phi_{1} d \approx l_{i}^{-1},
$$

by Lemma 4. Then $\Gamma_{s-m+2 i+1}^{\Delta} \sim-l_{0}^{-1} \cdots l_{i}^{-1}$ and so we take

$$
\Gamma_{s-m+2 i+2}=-\Gamma_{s-m+2 i}^{\Delta} / \Gamma_{s-m+2 i+1}^{\Delta} .
$$

Then

$$
\begin{aligned}
\Gamma_{s-m+2 i+2} & \sim e_{m-i-1}\left(l_{m}^{d} \phi_{1}\right) \cdots e_{1}\left(l_{m}^{d} \phi_{1}\right) l_{i+1}^{-1} \cdots l_{m-1}^{-1} l_{m}^{d-1} \phi_{1} d \\
& \approx e_{m-i-1}\left(l_{m}^{d} \phi_{1}\right),
\end{aligned}
$$

by Lemma 4 .

Thus by induction we can define $\Gamma_{s-m+1}, \ldots, \Gamma_{s+m-2}$ with the desired properties. If we take $i=m-1$ in (15) we get $\Gamma_{s+m-1} \approx l_{m-1}^{-1}$. We then set

$$
\Gamma_{s+m}=-\Gamma_{s+m-2}^{\Delta} /\left(d \Gamma_{s+m-1}^{\Delta}\right) \sim l_{m}^{d-1} \phi_{1} .
$$

If $d=1$, then $\Gamma_{s+m} \sim \phi_{1}$. Otherwise $\Gamma_{s+m} \approx l_{m}^{d-1}$ and hence $\Gamma_{s+m}^{\Delta} \sim$ $(d-1) l_{0}^{-1} \cdots l_{m}^{-1}$. Then

$$
\Gamma_{s+m}^{\Delta} /\left((d-1) \Gamma_{s+m-1}^{\Delta}\right) \sim-l_{m}^{-1} .
$$

We therefore take

$$
\Gamma_{s+m+1}=\Gamma_{s+m}\left(\Gamma_{s+m}^{\Delta} /(1-d) \Gamma_{s+m-1}^{\Delta}\right)^{d-1} \sim \phi_{1} .
$$

A routine check then establishes that (i)-(iv) hold in this case.

Case 1b: $d<1$ or $d=1$ and $\phi_{1} \rightarrow 0$. Here (11) and Lemma 4 give $\Gamma_{s-m} \approx$ $l_{0}^{-1}$. Hence $\Gamma_{s-m}^{\Delta} \sim-l_{0}^{-1}$ and so we take $\Gamma_{s-m+1}=-\Gamma_{s-m} / \Gamma_{s-m}^{\Delta}$. Again (11) and the lemma may be used to deduce that $\Gamma_{s-m+1} \approx e_{m}\left(l_{m}^{d} \phi_{1}\right)$.

Suppose that $0 \leq i<m-1$ and that $\Gamma_{s-m+2 i}$ and $\Gamma_{s-m+2 i+1}$ have been defined with $\Gamma_{s-m+2 i} \approx l_{i}^{-1}$ and $\Gamma_{s-m+2 i+1} \approx e_{m-i}\left(l_{m}^{d} \phi_{1}\right)$. 
We take

$$
\Gamma_{s-m+2 i+2}=-\Gamma_{s-m+2 i+1}^{\triangle} / \Gamma_{s-m+2 i}^{\Delta} .
$$

As before, $\Gamma_{s-m+2 i+2} \approx l_{i+1}^{-1}$ and we take

$$
\Gamma_{s-m+2 i+3}=-\Gamma_{s-m+2 i+1}^{\Delta} / \Gamma_{s-m+2 i+2}^{\Delta} \approx e_{m-i-1}\left(l_{m}^{d} \phi_{1}\right) .
$$

This accounts for the definitions of $\Gamma_{s-m+1}, \ldots, \Gamma_{s+m-1}$. Then we set

$$
\Gamma_{s+m}=-\Gamma_{s+m-1}^{\Delta} /\left(d \Gamma_{s+m-2}^{\triangle}\right) \sim l_{m}^{d-1} \phi_{1} .
$$

If $d=1$, then of course $\Gamma_{s+m} \sim \phi_{1}$. Otherwise, $\Gamma_{s+m} \approx l_{m}^{d-1}$ and we take

$$
\Gamma_{s+m+1}=\Gamma_{s+m}\left(\Gamma_{s+m}^{\Delta} /(1-d) \Gamma_{s+m-1}^{\triangle}\right)^{d-1} \sim \phi_{1} .
$$

Conditions (i)-(iv) are then satisfied.

Case 2: $m>s>0$. We take $\Gamma_{0}=\phi^{\Delta} \approx l_{0}^{-1}$ and for $i=1, \ldots, m-s-1$ we take $\Gamma_{i}=-\Gamma_{0} / \Gamma_{i-1}^{\Delta}$. Then

$$
\Gamma_{i} \sim e_{s-1}\left(l_{m}^{d} \phi_{1}\right) \cdots e_{1}\left(l_{m}^{d} \phi_{1}\right) l_{i}^{-1} \cdots l_{m-1}^{-1} l_{m}^{d-1} \phi_{1} d \approx l_{i}^{-1} \text {. }
$$

Case 2a: $d>1$ or $d=1$ and $\phi_{1} \rightarrow \infty$. In this case, Lemma 4 implies that $\gamma(\phi)>\gamma\left(l_{m-s}\right)$ and hence we take $\Gamma_{m-s}=\phi$ and $\Gamma_{m-s+1}=-\Gamma_{0} / \Gamma_{m-s-1}^{\Delta} \approx$ $l_{m-s}^{-1}$. Similarly we take $\Gamma_{m-s+2}=-\Gamma_{0} / \Gamma_{m-s+1}^{\Delta} \approx e_{s-1}\left(l_{m}^{d} \phi_{1}\right)$. Then for $i=1$, $\ldots, s-2$, we define

$$
\Gamma_{m-s+2 i+1}=-\Gamma_{m-s+2 i}^{\triangle} / \Gamma_{m-s+2 i-1}^{\triangle}
$$

and

$$
\Gamma_{m-s+2 i+2}=-\Gamma_{m-s+2 i}^{\triangle} / \Gamma_{m-s+2 i+1}^{\triangle}
$$

Then $\Gamma_{m-s+2 i+1} \approx l_{m-s+i}^{-1}$ and $\Gamma_{m-s+2 i+2} \approx e_{s-i-1}\left(l_{m}^{d} \phi_{1}\right)$. We define $\Gamma_{m+s-1}$ by taking $i=s-1$ in $(17)$, so that $\Gamma_{m+s-1} \approx l_{m-1}^{-1}$. Then we set

$$
\Gamma_{m+s}=-\Gamma_{m+s-2}^{\triangle} /\left(d \Gamma_{m+s-1}^{\triangle}\right) \sim l_{m}^{d-1} \phi_{1} .
$$

If $d \neq 1$, we take

$$
\Gamma_{m+s+1}=\Gamma_{m+s}\left(\Gamma_{m+s}^{\triangle} /(1-d) \Gamma_{m+s-1}^{\triangle}\right)^{d-1} \sim \phi_{1},
$$

and here too (i)-(iv) are satisfied.

Case 2b: $d<1$ or $d=1$ and $\phi_{1} \rightarrow 0$. Now $\gamma(\phi)<\gamma\left(l_{m-s}\right)$ and so we take $\Gamma_{m-s}=-\Gamma_{0} / \Gamma_{m-s-1}^{\Delta} \approx l_{m-s}^{-1}$ and $\Gamma_{m-s+1}=\phi$. Then for $i=1, \ldots, s-1$, we take

and

$$
\Gamma_{m-s+2 i}=-\Gamma_{m-s+2 i-1}^{\triangle} / \Gamma_{m-s+2 i-2}^{\triangle}
$$

Then let

$$
\Gamma_{m-s+2 i+1}=-\Gamma_{m-s+2 i-1}^{\triangle} / \Gamma_{m-s+2 i}^{\triangle}
$$

$$
\Gamma_{m+s}=-\Gamma_{m+s-1}^{\triangle} /\left(d \Gamma_{m+s-2}^{\triangle}\right) \sim l_{m}^{d-1} \phi
$$

and, if $d \neq 1$, let

$$
\Gamma_{m+s+1}=\Gamma_{m+s}\left(\Gamma_{m+s}^{\triangle} /(1-d) \Gamma_{m+s-1}^{\triangle}\right)^{d-1} \sim \phi_{1} .
$$

One checks (i)-(iv) as before. This completes the proof of Theorem 6 . 
Theorem 6 allows us to strengthen Theorem 5 in two ways. First, the existence of the $\Gamma_{i}$ 's implies a rank bound. Second, provided $\nu\left(\phi_{1}\right) \neq 0$, we can treat $\phi_{1}$ in the same way as we did $\phi$. If $\phi_{1}$ tends to a nonzero finite limit, then it is trivial that $\mathscr{F}$ contains elements asymptotic to $\phi_{1}$ since $\mathbf{R} \subset \mathscr{F}$, but this fact gives us no information about the asymptotic behaviour of $\phi_{1}^{\prime}$ (beyond the fact that $\phi_{1}^{\prime} \rightarrow 0$ ).

For $f$ an element of a Hardy field and $s$ an integer, define $E_{s}(f)$ to be $e_{s}(f)$ if $s \geq 0$ and $\left(e_{-s}(f)\right)^{-1}$ if $s<0$. Let $\phi$ be an element of a Hardy field with $\nu(\phi) \neq 0$. A nested form for $\phi$ will be a finite sequence $\left\{\left(s_{i}, m_{i}, d_{i}\right.\right.$, $\left.\left.\phi_{i}\right), i=1, \ldots, k\right\}$ with the following properties:

(a) For each $i, s_{i}$ is an integer, $m_{i}$ a nonnegative integer, $d_{i}$ a positive real number, and $\phi_{i}$ an element of a Hardy field.

(b) $\phi=E_{s_{1}}\left(l_{m_{1}}^{d_{1}} \phi_{1}\right)$ and $\phi_{i-1}=E_{s_{i}}\left(l_{m_{i}}^{d_{i}} \phi_{i}\right)$ for $i=2, \ldots k$,

(c) $\gamma\left(\phi_{i}\right)<\gamma\left(l_{m_{i}}\right)$ for $i=1, \ldots, k$.

(d) $\nu\left(\phi_{k}\right)=0$.

(e) $d_{k} \neq 1$ unless $s_{k}=0$ or $m_{k}=0$.

The following is an easy consequence of the definition and Lemma 4.

Proposition 2. Let $\left\{\left(s_{i}, m_{i}, d_{i}, \phi_{i}\right), i=1, \ldots, k\right\}$ be a nested form for some $\phi$. Then for $i=1, \ldots, k-1$,

$$
m_{i+1} \geq \max \left\{m_{i}+1, m_{i}+s_{i+1}\right\}
$$

and if $m_{i+1}=m_{i}+s_{i+1}$, then $d_{i+1} \leq 1$.

The next result gives the promised strengthening of Theorem 5 .

Theorem 7. Let $\mathscr{F}$ be a Rosenlicht field of rank $r$. Let $\phi$ be a positive element of $\mathscr{F}$ with $\nu(\phi) \neq 0$. Then $\phi$ has a nested form $\left\{\left(s_{i}, m_{i}, d_{i}, \phi_{i}\right), 1 \leq i \leq k\right\}$ such that

$$
\sum_{j=1}^{k}\left|s_{j}\right|+\delta_{k}+m_{k} \leq r,
$$

where $\delta_{k}=0$ if $d_{k}=1$ and $m_{k}=0$, and $\delta_{k}=1$ otherwise. Moreover, there exist elements $\Gamma_{0}, \ldots, \Gamma_{\Sigma}$ of $\mathscr{F}$ such that the following properties hold:

(i) $\Sigma=\sum_{j=1}^{k}\left|s_{j}\right|+\delta_{k}+m_{k}$.

(ii) Each $\Gamma_{i}$ may be expressed as a rational function of a finite set of real powers of $\phi, \phi^{\prime}, \ldots, \phi^{(i)}$ with real coefficients. Conversely, $\phi^{(i)}$ may be similarly expressed as a rational function of a finite set of real powers of $\Gamma_{0}, \Gamma_{1}, \ldots, \Gamma_{i}$. Both of these rational functions are computable.

(iii) For $i=0, \ldots, \Sigma-1$, we have $\gamma\left(\Gamma_{i}\right)>\gamma\left(\Gamma_{i+1}\right)$. Also $\gamma\left(\Gamma_{\Sigma}\right)=\gamma(1)$.

(iv) The set of comparability classes of the $\Gamma_{i}$ 's is equal to

$$
\gamma(1) \cup \bigcup_{i=0}^{m_{k}+\delta_{k}-1} \gamma\left(l_{i}\right) \cup \bigcup_{j=1}^{k} \bigcup_{t=1}^{\left|s_{j}\right|} \gamma\left(e_{t}\left(l_{m_{j}}^{d_{j}} \phi_{j}\right)\right) .
$$

Of course if $\phi \in \mathscr{F}^{*}$ with $\nu(\phi)=0$, then Theorem 7 may be applied to $\pm(\phi-\lim \phi)$. We call $\Sigma$ the length of the nested form. 
Proof of Theorem 7. For $j=1, \ldots, k$, we define $\delta_{j}$ by $\delta_{j}=0$ if $d_{j}=1$ and $m_{j}=0$, and $\delta_{j}=1$ otherwise. We write $t_{j}=\left|s_{j}\right|+\delta_{j}$. By Theorem 5 , $s_{1}, m_{1}, d_{1}$, and $\phi_{1}$ exist such that $\phi=E_{s_{1}}\left(l_{m_{1}}^{d_{1}} \phi_{1}\right)$. If $\nu\left(\phi_{1}\right)=0$, then $\left\{\left(s_{1}\right.\right.$, $\left.\left.m_{1}, d_{1}, \phi_{1}\right)\right\}$ is a nested form for $\phi$, as conditions (c) and (e) of the definition are given by (ii) and (iii) of Theorem 5. Theorem 6 then implies the existence of $\Gamma_{0}, \ldots, \Gamma_{t_{1}+m_{1}}$ with suitable properties.

If $\nu\left(\phi_{1}\right) \neq 0$, we may apply Theorem 5 to either $\Gamma_{t_{1}+m_{1}}$ or $\Gamma_{t_{1}+m_{1}}^{-1}$ according to whether $\nu\left(\Gamma_{t_{1}+m_{1}}\right)<0$ or not. Thus $\Gamma_{t_{1}+m_{1}}=E_{s_{2}}\left(l_{m_{2}}^{d_{2}} \psi_{2}\right)$ for suitable choices of $s_{2}, m_{2}, d_{2}$, and $\psi_{2}$. Theorem 6 then guarantees the existence of elements $\Gamma_{0}^{1}, \ldots, \Gamma_{\sigma_{1}}^{1}$ of $\mathscr{F}$ satisfying (i)-(iv) of that theorem. The comparability classes of these elements will include $\gamma\left(l_{0}\right), \ldots, \gamma\left(l_{m_{1}+\delta_{1}-1}\right)$ but will otherwise be distinct from the comparability classes of the $\Gamma_{i}$ 's, since $\gamma\left(\phi_{1}\right)<\gamma\left(l_{m_{1}}\right)$. Hence we may relabel $\Gamma_{m_{1}+\delta_{1}}^{1}, \ldots, \Gamma_{\sigma_{1}}^{1}$ as $\Gamma_{t_{1}+m_{1}+1}, \ldots, \Gamma_{\left|s_{1}\right|+t_{2}+m_{2}}$ and then $\gamma\left(\Gamma_{0}\right)>\gamma\left(\Gamma_{1}\right)>\cdots>\gamma\left(\Gamma_{\left|s_{1}\right|+t_{2}+m_{2}}\right)$. Since $\Gamma_{t_{1}+m_{1}} \sim \phi_{1}$, there is a $\phi_{2} \sim \psi_{2}$ such that $\phi_{1}=E_{s_{2}}\left(l_{m}^{d_{2}} \phi_{2}\right)$. If $\nu\left(\phi_{2}\right)=0$, then $\left\{\left(s_{1}, m_{1}, d_{1}, \phi_{1}\right),\left(s_{2}, m_{2}\right.\right.$, $\left.\left.d_{2}, \phi_{2}\right)\right\}$ is a nested form for $\phi$ and properties (i)-(iv) of the statement of the theorem are easily seen to hold. Otherwise we apply Theorem 5 to $\Gamma_{\left|s_{1}\right|+t_{2}+m_{2}}$ and continue as above. In this way we obtain a sequence $\left\{\left(s_{j}, m_{j}, d_{j}, \phi_{j}\right)\right.$, $j=1, \ldots, k\}$ and the process will continue until some $\nu\left(\phi_{k}\right)$ is zero. It is clear that this must happen after a finite number of steps since (18) must continue to hold and $\left\{m_{j}\right\}$ is strictly increasing; moreover, $t_{j} \geq 1$. So $\phi$ has a nested form. The inequality (18), the existence of the $\Gamma_{j}$ 's, and properties (i)-(iv) are then clear from Theorem 6 and the above method of construction of the nested form. This completes the proof of Theorem 7 .

\section{AN APPLICATION TO ALGEBRAIC DIFFERENTIAL EQUATIONS}

Suppose that $y$ satisfies an algebraic differential equation of order $r$ over $\mathbf{R}$. If $\mathbf{R}\left(y, y^{\prime}, \ldots, y^{(r)}\right)$ is a Hardy field, then its rational rank is no greater than $r$ and hence, by Theorem 4, it is contained in a Rosenlicht field $\mathscr{F}$ of rank $\leq r$. If $y$ is positive and $\nu(y) \neq 0$, then $y$ must possess a nested form as given by Theorem 7 . In other cases the theorem may be applied to $-y$ or to $\pm(y-\lim y)$ as appropriate. (We do not consider here the case when $y$ is constant.)

For small values of $r,(18)$ constitutes a severe restriction on the possible nested forms and it becomes feasible to consider possible cases individually. Let $\phi$ be a nested form of length $\Sigma$, where $\Sigma \leq r$, and consider first the case when $\Sigma=r$. Then we may define $\Gamma_{0}, \ldots, \Gamma_{r}$ as in Theorem 7 . We write $\phi$ and its derivatives in terms of the $\Gamma_{i}$ 's and substitute into the differential equation. We refer to the equation thus obtained as the $\Gamma$-equation. In the $\Gamma$ equation, $\gamma\left(\Gamma_{r}\right)=\gamma(1)$ but otherwise the comparability classes of the $\Gamma_{i}$ 's are distinct from each other and from the comparability class of the constants. So, in particular, the coefficient of the leading product of $\Gamma_{0}, \ldots, \Gamma_{r-1}$ must be asymptotically zero, which implies a condition on the parameters of the nested form. If this condition is unsatisfiable, then the particular nested form under consideration cannot occur.

Next suppose that $\Sigma<r$. Then we define $\Gamma_{0}, \ldots, \Gamma_{\Sigma-1}$ as in Theorem 7. An inspection of the proof of Theorem 6 shows that in all cases, either 
$\Gamma_{\Sigma-1} \sim A l_{m_{k}}^{d_{k}}$ or $\Gamma_{\Sigma-1} \sim A l_{m_{k}}^{d_{k}-1}$, where $A$ is some nonzero constant. In the present situation $d_{k}$ will be a parameter to be determined, so these cases may be treated similarly to each other. We relabel $\Gamma_{\Sigma-1}$ as $\widetilde{\Gamma}_{\Sigma-1}$ and define $\widetilde{\Gamma}_{\Sigma}$, $\ldots, \widetilde{\Gamma}_{r}$ as follows. Let $\zeta$ denote an element of the Rosenlicht field generated by $\phi$ which is asymptotic to $-l_{0} l_{1} \cdots l_{m_{k}-1}$. By Theorem 6 , we can easily give such an element in terms of $\Gamma_{0}, \ldots, \Gamma_{\Sigma-2}$. Then for $i=0, \ldots, r-\Sigma$, we define $\widetilde{\Gamma}_{\Sigma+i}=\zeta\left(\widetilde{\Gamma}_{\Sigma+i-1}\right)^{\prime}$. Then (for the case when $\Gamma_{\Sigma-1} \sim A l_{m_{k}}^{d_{k}}$ )

$$
\widetilde{\Gamma}_{\Sigma+i} \sim A d_{k}\left(d_{k}-1\right) \cdots\left(d_{k}-i-1\right) l_{m_{k}}^{d_{k}-i} .
$$

We can then rewrite $\phi, \ldots, \phi^{(r)}$ in terms of $\Gamma_{0}, \Gamma_{1}, \ldots, \Gamma_{\Sigma-1}, \widetilde{\Gamma}_{\Sigma}, \ldots, \widetilde{\Gamma}_{r}$ and substitute into the differential equation for $y$. Then we can use the fact that $\gamma\left(\Gamma_{0}\right), \ldots, \gamma\left(\Gamma_{\Sigma-2}\right), \gamma\left(\widetilde{\Gamma}_{\Sigma-1}\right)$ are all different, and the known asymptotic forms of $\widetilde{\Gamma}_{\Sigma-1}, \ldots, \widetilde{\Gamma}_{r}$, to obtain equations for the parameters of the nested form. As before, these may in some cases be unsatisfiable, leading to the conclusion that the nested form under consideration cannot occur for solutions of the given differential equation. For some equations, all the possible nested forms may be eliminated, giving the conclusion that the equation has no (nonconstant) solutions lying in a Hardy field.

We next illustrate the above argument by looking in greater detail at secondorder equations over R. Suppose then that $\phi$ satisfies such an equation. Since $\delta_{k}=1$ unless $m_{k}=0$ and, by Proposition $2, m_{i} \geq i-1$, the inequality (18) implies that $k \leq 2$. Possibilities are therefore $\left\{k=2, s_{1}=s_{2}=0, m_{1}=0\right.$, $\left.m_{2}=1\right\}$ or $\left\{k=1, s_{1}=0, m_{1} \leq 1\right\}$ or $\left\{k=1, s_{1} \leq 2, m_{1}=0\right\}$. Note in particular that we cannot have $k=s_{1}=m_{1}=1$ since then $\delta_{1}=1$ and (18) will be violated. Moreover, the case $k=1, s_{1}=2, m_{1}=0$ is only possible if $d_{1}=1$. Let $A$ stand for a nonzero constant and $C$ for a constant. The possible asymptotic forms for $\phi$ are then:

(I) $C+x^{d_{1}} l_{1}^{d_{2}}(A+\varepsilon)$ with $C=0$ if $d_{1}>0$ or if $d_{1}=0$ and $d_{2}>0$;

(II) $C \pm e_{1}\left(x^{d_{1}}(A+\varepsilon)\right)$ with $C=0$ if $A>0$;

(III) $\pm e_{2}(x(A+\varepsilon))$ with $A>0$;

(IV) $C \pm E_{-2}(x(A+\varepsilon))$ with $A>0$. We calculate the $\Gamma_{i}$ 's in a selection of cases. Full details are to be found in [11].

Case (I)(i): $\phi=C+x^{d_{1}} l_{1}^{d_{2}}(A+\varepsilon), d_{1} \neq 0, d_{2} \neq 0$. We take $\Gamma_{0}=\phi-C$ and

$$
\Gamma_{1}=\Gamma_{0}\left(\Gamma_{0}^{\triangle} / d_{1}\right)^{d_{1}} \sim l_{1}^{d_{2}} A \text {. }
$$

Then, as in Theorem 6,

$$
\Gamma_{2}=\Gamma_{1}\left(d_{1} \Gamma_{1}^{\triangle} / d_{2} \Gamma_{0}^{\Delta}\right)^{d_{2}} \sim A .
$$

Next, we calculate $\phi, \phi^{\prime}$, and $\phi^{\prime \prime}$ in terms of $\Gamma_{0}, \Gamma_{1}$, and $\Gamma_{2}$. Of course

$$
\phi=\Gamma_{0}+C,
$$

and therefore $\phi^{\prime}=\Gamma_{0}^{\prime}$. Hence from (19) (using the fact that $\Gamma_{0}^{\Delta}=\Gamma_{0}^{\prime} / \Gamma_{0}$ ), we have

$$
\phi^{\prime}=d_{1} \Gamma_{0}^{1-1 / d_{1}} \Gamma_{1}^{1 / d_{1}}
$$


Differentiating (22) gives

$$
\phi^{\prime \prime}=\left(d_{1}-1\right)\left(\Gamma_{1} / \Gamma_{0}\right)^{1 / d_{1}} \Gamma_{0}^{\prime}+\left(\Gamma_{1} / \Gamma_{0}\right)^{1 / d_{1}-1} \Gamma_{1}^{\prime},
$$

and then substituting for $\Gamma_{0}^{\prime}$ and $\Gamma_{1}^{\prime}$ from (19) and (20) gives

$$
\phi^{\prime \prime}=d_{1}\left(d_{1}-1\right) \Gamma_{0}^{1-2 / d_{1}} \Gamma_{1}^{2 / d_{1}}+d_{2} \Gamma_{0}^{1-2 / d_{1}} \Gamma_{1}^{2 / d_{1}-1 / d_{2}} \Gamma_{2}^{1 / d_{2}}
$$

Case (I)(ii): $\phi=C+x^{d_{1}}(A+\varepsilon), d_{1} \neq 0$. Here the rank of $\mathbf{R}\langle\phi\rangle$ (i.e. $\left.\mathbf{R}\left(\phi, \phi^{\prime}, \phi^{\prime \prime}, \ldots\right)\right)$ is equal to 1 . Accordingly, we define $\widetilde{\Gamma}_{0}=\phi-C, \widetilde{\Gamma}_{1}=\widetilde{\Gamma}_{0}^{\prime}$, and $\widetilde{\Gamma}_{2}=\widetilde{\Gamma}_{1}^{\prime}$.

Case (I)(iii): $\phi=C+l_{1}^{d_{2}}(A+\varepsilon), d_{2} \neq 0$. Now an analysis similar to the above leads to the formulae

$$
\begin{gathered}
\phi=C+\Gamma_{2} \Gamma_{1}^{-d_{2}}, \\
\phi^{\prime}=d_{2} \Gamma_{0}(\phi-C)=d_{2} \Gamma_{0} \Gamma_{1}^{-d_{2}} \Gamma_{2},
\end{gathered}
$$

and

$$
\phi^{\prime \prime}=d_{2}^{2} \Gamma_{0}^{2} \Gamma_{1}^{-d_{2}} \Gamma_{2}-d_{2} \Gamma_{0}^{2} \Gamma_{1}^{-1-d_{2}} \Gamma_{2}
$$

Case (II): $\phi=C \pm \rho_{1}\left(x^{d_{1}}(A+\varepsilon)\right), d_{1}>0$. The case when $d_{1}=1$ is similar to (I)(ii). For $d_{1} \neq 1$, the equations for $\phi$ and its derivatives are

$$
\phi= \pm \Gamma_{0}+C, \quad \phi^{\prime}= \pm d_{1} \Gamma_{0} \Gamma_{1},
$$

and

$$
\phi^{\prime \prime}= \pm\left(d_{1}^{2} \Gamma_{0} \Gamma_{1}^{2}+d_{1}\left(d_{1}-1\right) \Gamma_{0} \Gamma_{1}\left(\frac{\Gamma_{2}}{\Gamma_{1}}\right)^{\frac{1}{d_{1}-1}}\right)
$$

It is understood that one must have a consistent choice of + or - sign in these equations.

Similar formulae hold in Cases (III) and (IV).

5.1 Examples. We apply the results of the above analysis to the equation $\phi^{\prime \prime}+\lambda \phi=0, \lambda \in \mathbf{R}$. Of course, the solutions of this equation are exceedingly well known, but it nonetheless illustrates the method quite well.

We start with Case (I)(i) and substitute (21) and (23) into the differential equation. This gives us the $\Gamma$-equation

$$
d_{1}\left(d_{1}-1\right) \Gamma_{0}^{1-2 / d_{1}} \Gamma_{1}^{2 / d_{1}}+d_{2} \Gamma_{0}^{1-2 / d_{1}} \Gamma_{1}^{2 / d_{1}-1 / d_{2}} \Gamma_{2}^{1 / d_{2}}+\lambda\left(\Gamma_{0}+C\right)=0 .
$$

If $d_{1}>0$, then $C=0$ and the leading term is $\lambda \Gamma_{0}$. Hence $\lambda=0$. If $d_{1} \neq 1$, the leading term becomes $d_{1}\left(d_{1}-1\right) \Gamma_{0}^{1-2 / d_{1}} \Gamma_{1}^{2 / d_{1}}\left(\right.$ since $\left.\Gamma_{1}^{-1 / d_{2}} \sim l_{1}^{-1}\right)$. If $d_{1}=1$, the only remaining term is $d_{2} \Gamma_{0}^{-1} \Gamma_{1}^{2-1 / d_{2}} \Gamma_{2}^{1 / d_{2}}$. In neither case can (29) be satisfied.

Similarly, if $d_{1}<0$ the leading term is $\lambda C$ which must therefore be zero. The next largest term is $\lambda \Gamma_{0}$ and hence $\lambda=0$. The leading term then becomes again $d_{1}\left(d_{1}-1\right) \Gamma_{0}^{1-2 / d_{1}} \Gamma_{1}^{2 / d_{1}}$ and as before the $\Gamma$-equation cannot be satisfied. Thus Case (I)(i) cannot occur. 
In Case (I)(ii), the rank, $r$, of $\mathbf{R}\langle\phi\rangle$ is one less than the order of the differential equation. Hence we work with $\widetilde{\Gamma}_{0}, \ldots, \widetilde{\Gamma}_{r}$. Here $r=1$ and $\widetilde{\Gamma}_{0}=\phi-C=x^{d_{1}}(A+\varepsilon)$. Thus $\widetilde{\Gamma}_{1}=\Gamma_{0}^{\prime}=d_{1} x^{d_{1}-1}(A+\varepsilon) \sim d_{1} x^{-1} \Gamma_{0}$ and $\widetilde{\Gamma}_{2}=\tilde{\Gamma}_{1}^{\prime} \sim d_{1}\left(d_{1}-1\right) x^{-2} \Gamma_{0}$. On substituting into the differential equation, we obtain

$$
\widetilde{\Gamma}_{2}+\lambda\left(\widetilde{\Gamma}_{0}+C\right)=0
$$

On using the fact that $\widetilde{\Gamma}_{2} \sim d_{1}\left(d_{1}-1\right) x^{-2} \widetilde{\Gamma}_{0}$, we see that $\lambda=0$ and hence $d_{1}\left(d_{1}-1\right) x^{-2} \widetilde{\Gamma}_{0}=0$. This is possible if $d_{1}=1$. So we obtain a possible asymptotic form for $\phi$ when $\lambda=0$, namely $\phi=x(A+\varepsilon)$.

Similar calculations to those in I(i) show that Case (I)(iii) cannot occur (see [11]).

Now for Case (II). When $d_{1} \neq 1$, the $\Gamma$-equation is

$$
d_{1}^{2} \Gamma_{0} \Gamma_{1}^{2}+d_{1}\left(d_{1}-1\right) \Gamma_{0} \Gamma_{1}^{1-\frac{1}{d_{1}-1}} \Gamma_{2}^{\frac{1}{d_{1}-1}}+\lambda\left(\Gamma_{0}+C\right)=0 .
$$

The leading term is $d_{1}^{2} \Gamma_{0} \Gamma_{1}^{2}$ if $d_{1}>1$ or if $\lambda=0$, and $\lambda \Gamma_{0}$ otherwise, since $\Gamma_{1} \sim A x^{d_{1}-1}$. In neither case can the leading coefficient be asymptotically zero. Thus $(31)$ is unsatisfiable.

If $d_{1}=1$, we work with $\Gamma_{0}$ and its derivatives. We have $\widetilde{\Gamma}_{1}=\widetilde{\Gamma}_{0}^{\prime} \sim A \Gamma_{0}$ and $\widetilde{\Gamma}_{2}=\Gamma_{0}^{\prime \prime} \sim A^{2} \Gamma_{0}$. The $\Gamma$-equation is then

$$
A^{2} \Gamma_{0}+\lambda\left(\Gamma_{0}+C\right) \sim 0 .
$$

If $A$ is negative, we see that $\Gamma_{0} \rightarrow 0$ and hence $C$ is forced to be zero. Thus, whatever the sign of $A$, we require $A^{2}+\lambda=0$. This is impossible if $\lambda \geq 0$ but if $\lambda<0$ we obtain two possible asymptotic forms for $\phi$, namely $e_{1}(x(\sqrt{-} \lambda+\varepsilon))$ and $e_{1}(-(x(\sqrt{-\lambda}+\varepsilon)))$.

Similar calculations to those already given show that Cases (III) and (IV) cannot occur. We have thus rediscovered the following facts:

(a) If $\lambda>0, \phi^{\prime \prime}+\lambda \phi$ has no nonconstant solutions lying in a Hardy field.

(b) If $\lambda=0$, the possible asymptotic form for $\phi$ is $x(A+\varepsilon)$.

(c) If $\lambda<0$, two possible asymptotic forms are $e_{1}(x(\sqrt{-} \lambda+\varepsilon))$ and $e_{1}(-x(\sqrt{-} \lambda+\varepsilon))$.

Finally we take a brief look at how the techniques apply to the soliton equation

$$
\phi^{\prime \prime}+\phi+\lambda \phi^{3}=0
$$

We may assume that $\lambda \neq 0$. Cases (I), (III), and (IV) are easily eliminated. For Case (II), when $d_{1} \neq 1$, we substitute (27) and (28) into (33). This gives

$$
d_{1}^{2} \Gamma_{0} \Gamma_{1}^{2}+d_{1}\left(d_{1}-1\right) \Gamma_{0} \Gamma_{1}^{1-\frac{1}{d_{1}-1}} \Gamma_{2}^{\frac{1}{d_{1}-1}}+\Gamma_{0}+C+\lambda\left(\Gamma_{0}+C\right)^{3}=0,
$$

where we have absorbed the \pm into $C$.

Suppose first that $C \neq 0$ so that $A<0$. In that case, we must have $\lambda=$ $-1 / C^{2}$ and then $\Gamma_{0}$ is a factor. Hence

$$
d_{1}^{2} \Gamma_{1}^{2}+d_{1}\left(d_{1}-1\right) \Gamma_{1}^{1-\frac{1}{d_{1}-1}} \Gamma_{2}^{\frac{1}{d_{1}-1}}-2-3 \Gamma_{0} / C-\Gamma_{0}^{2} / C^{2}=0 .
$$


If $d_{1}>1$ then $\Gamma_{1} \rightarrow \infty$ and $1-\frac{1}{d_{1}-1}<1$. But then the term $d_{1}^{2} \Gamma_{1}^{2}$ dominates the left-hand side of (35), and so this case cannot occur. If $d_{1}<1$, then $\Gamma_{1} \rightarrow 0$ and (35) is impossible since all terms except ' 2 ' tend to zero. If $C=0$ and $A<0$, a similar analysis shows that (34) cannot be satisfied, while, if $A>0$, the term $\Gamma_{0}^{3}$ dominates (34), again giving a contradiction.

Consider now the case when $d_{1}=1$. Here we take $\widetilde{\Gamma}_{1}=\Gamma_{0}^{\prime}$ and $\widetilde{\Gamma}_{2}=\widetilde{\Gamma}_{1}^{\prime}$, so that $\widetilde{\Gamma}_{2} \sim A^{2} \Gamma_{0}$. The equation (33) then yields

$$
A^{2} \Gamma_{0}+\Gamma_{0}+C+\lambda\left(\Gamma_{0}+C\right)^{3}=o\left(\Gamma_{0}\right) .
$$

If $C \neq 0$, then as before $\lambda=-1 / C^{2}$ and the coefficient of $\Gamma_{0}$ on the lefthand side of (36) is $A^{2}-2$. So $A=-\sqrt{2}$ and, provided $\lambda<0$, a possible asymptotic form for the solution of (33) is

$$
\frac{ \pm 1}{\sqrt{-\lambda}} \pm e^{x(-\sqrt{2}+\varepsilon)}
$$

If $C=0$ and $A<0$, then $\left(A^{2}+1\right) \Gamma_{0}$ dominates the left-hand side of (36), which is not possible, and, if $A>0$, then $\lambda \Gamma_{0}^{3}$ similarly dominates (36). Thus we have the conclusion that if $\lambda>0$, no solution of the soliton equation (33) can lie in a Hardy field, while for $\lambda<0$ a possible asymptotic form is given by (37).

\section{REFERENCES}

1. M. Boshernitzan, An extension of Hardy's class L of 'orders of infinity', J. Analyse Math. 39 (1981), 235-255.

2. - Hardy fields, existence of transexponential functions and the hypertranscendence of solution to $g(g(x))=e^{x}$, Aequationes Math. 30 (1986), 258-280.

3. N. Bourbaki, Fonctions d'une variable réele, Ch. V, 2nd ed. Hermann, Paris, 1961.

4. P. du Bois-Reymond, Ueber asymptotische Werthe, infinitäre Approximationen und infinitäre Auflösung von Gleichungen, Math. Ann. 8 (1875), 362-414.

5. G. H. Hardy, Orders of infinity, Cambridge Univ. Press, Cambridge, 1910.

6. M. Rosenlicht, Growth properties of functions in Hardy fields, Trans. Amer. Math. Soc. 299 (1987), 261-272.

7. — Hardy fields, J. Math. Anal. Appl. 93 (1983), 297-311.

8. __ Rank change on adjoining real powers to Hardy fields, Trans. Amer. Math. Soc. 284 (1984), 829-836.

9. — The rank of a Hardy field, Trans. Amer. Math. Soc. 280 (1983), 659-671.

10. J. R. Shackell, Growth estimates for Exp-Log functions, J. Symbolic Comp. 10 (1990), 611 632.

11. _ Rosenlicht fields and asymptotic forms, Technical Report, University of Kent at Canterbury, 1990.

Institute of Mathematics, The University of Canterbury, Kent CT2 7NF, United KINGDOM

E-mail address: jrs@ukc.ac.uk 